\title{
Cortistatin exerts antiproliferation and antimigration effects in vascular smooth muscle cells stimulated by Ang II through suppressing ERK1/2, p38 MAPK, JNK and ERK5 signaling pathways
}

\author{
Ying Wang ${ }^{1}$, Xin Zhang ${ }^{1}$, Lei Gao ${ }^{1}$, Jihe $\mathrm{Li}^{1}$, Wenjia Chen ${ }^{1}$, Jinyu Chi ${ }^{1}$, Xiaohui Zhang ${ }^{1}$, Yu Fu ${ }^{1}$, \\ Meng Zhao ${ }^{1}$, Na Liu ${ }^{1}$, Yang Li ${ }^{1}$, Yang $\mathrm{Xu}^{1}$, Kelaier Yang ${ }^{2}$, Xinhua Yin ${ }^{1}$, Yue Liu ${ }^{1}$ \\ ${ }^{1}$ Department of Cardiology, ${ }^{2}$ Department of Endocrine, the First Affiliated Hospital of Harbin Medical University, Harbin 150001, China \\ Contributions: (I) Conception and design: Y Wang, X Yin, Y Liu; (II) Administrative support: X Yin, Y Liu, X Zhang, J Chi; (III) Provision of study \\ materials or patients: Y Wang; (IV) Collection and assembly of data: Y Wang, L Gao; (V) Data analysis and interpretation: Y Wang, W Chen; (VI) \\ Manuscript writing: All authors; (VII) Final approval of manuscript: All authors. \\ Correspondence to: Xinhua Yin; Yue Liu. Department of Cardiology, the First Affiliated Hospital of Harbin Medical University, No. 23, YouZheng \\ Street, NanGang District, Harbin 150001, China. Email: yinxinhua5063@163.com; liuyue198002@163.com.
}

Background: Vascular remodeling, that contributes to cardiovascular diseases such as hypertension
develops by anomalous proliferation and migration of vascular smooth muscle cells (VSMCs). Cortistatin
(CST), a newly discovered biological peptide, has been acknowledged for its protective effects against
cardiovascular diseases. Whether CST has an inhibitory regulation role in angiotensin II (Ang II)-induced
proliferation and migration of VSMCs and what molecular mechanisms may participate in the CST
inhibition process are still unknown. Methods: VSMCs were divided into control group, Ang II $\left(10^{-7} \mathrm{M}\right)$ group, Ang II + PD98059 $\left(5 \times 10^{-5} \mathrm{M}\right)$ group, Ang II + SB203580 (10 $\left.{ }^{-5} \mathrm{M}\right)$ group, Ang II + SP600125 $\left(10^{-5} \mathrm{M}\right)$ group, Ang II + XMD17-109 $\left(10^{-6} \mathrm{M}\right)$ group, Ang II + CST $\left(10^{-8} \mathrm{M}\right)$ group and Ang II + CST $\left(10^{-7} \mathrm{M}\right)$ group. Cell proliferation was detected by western blotting and cell counting kit-8 (CCK8) analysis. Migration of VSMCs was measured by Transwell assay.

Results: Compared with control group, Ang II upregulated the expression levels of proliferating cell nuclear antigen (PCNA) and osteopontin (OPN) and downregulated that of $\alpha$-smooth muscle actin ( $\alpha$-SMA), increased the proliferation rate as shown by CCK8 and VSMC migration as shown by Transwell assay in cultured VSMCs of the Ang II group. Meanwhile, in Ang II-cultured VSMCs, we found activation of extracellular signal-regulated kinase (ERK) 1/2, p38 MAP kinase (p38 MAPK), c-Jun N-terminal kinase (JNK), and ERK5 pathways by western blotting at different time points. However, the proliferation and migration stimulated by Ang II were partly reversed by drug inhibitors of the four pathways, namely, PD98059, SB203580, SP600125 and XMD17-109. When Ang II-stimulated VSMCs were cultured with CST pretreatment, we found that proliferation and migration were greatly suppressed as well as that the ERK1/2, p38 MAPK, JNK and ERK5 pathways were deactivated by CST.

Conclusions: The accumulated data suggest that CST may play a protective role in Ang II-promoted proliferation and migration of VSMCs via inhibiting the mitogen-activated protein kinase (MAPK) family pathways, providing a new orientation of CST in protecting against cardiovascular diseases.

Keywords: Cortistatin (CST); angiotensin II (Ang II); vascular smooth muscle cells (VSMCs); proliferation; migration; mitogen-activated protein kinases (MAPK)

Submitted Jun 21, 2019. Accepted for publication Aug 26, 2019.

doi: $10.21037 /$ atm.2019.09.45

View this article at: http://dx.doi.org/10.21037/atm.2019.09.45 


\section{Introduction}

Hypertension is a pathological change in multiple organs, such as the heart, blood vessels, brain, and kidney, characterized by long-term, chronic, complex progress caused by genetics, the environment and neurohumoral/ hormonal drive. Angiotensin II (Ang II) comes from the renin-angiotensin system existing in the systemic and local circulation, constricts blood vessels through its structural and functional effects, and plays a critical role in the regulation of blood pressure and the pathogenesis of hypertension. Acute stimulation of Ang II can modulate salt/water balance, vasomotor tone and blood pressure in the body, while chronic stimulation can accelerate the proliferation and hypertrophy of vascular smooth muscle cells (VSMCs), influence VSMC migration and extracellular matrix deposition and result in cardiac and vascular remodeling $(1,2)$.

VSMCs are the predominant structure of the vascular system and play a pivotal role in maintaining blood pressure and angiostasis (3). Uncontrolled proliferation and migration of VSMCs is a key link in assorted cardiovascular diseases (hypertension, coronary heart disease, vascular restenosis and atherosclerosis) $(1,4)$. Ang II plays countless roles in regulating cardiovascular physiology and pathology by modulating different signaling pathways of VSMCs and by influencing the interactions between VSMCs and extracellular matrix, further leading to proliferation, contraction, migration, hypertrophy, fibrosis, rearrangement and lipid oxidation of VSMCs, which ultimately result in hypertension, atherosclerosis, restenosis and so on (2).

There are many signaling pathways involved in Ang II-mediated proliferation and migration of VSMCs, among which mitogen-activated protein kinase (MAPK) signaling pathways take priority in regulating cell proliferation, differentiation, migration and survival. The MAPK signaling pathways consist of mainly the extracellular signal-regulated kinase (ERK) 1/2, p38 MAP kinase (p38 MAPK), c-Jun N-terminal kinase (JNK), and ERK5 signaling pathways. A growing body of research has supported that Ang II triggers MAPK signaling cascades, including ERK1/2, p38 MAPK, JNK and ERK5 signaling pathways, which demonstrably take part in the differentiation, proliferation, migration, and fibrosis of VSMCs $(2,5,6)$. A number of studies have demonstrated that Ang II leads to VSMC proliferation and migration via activation of ERK1/2 signaling pathway (6-10). JNK has been reported as a participator in not only VSMC proliferation and migration $(1,10)$ but also cell stress $(2,11)$, apoptosis and inflammation (12). A previous study demonstrated that the p38 MAPK is also involved in Ang II-induced VSMC proliferation (10) and hypertrophy, which may be related to reactive oxygen species $(2,5)$. There have been fewer prior studies concerning ERK5 than there have been for other MAPK signaling pathways, as ERK5 is the most recently discovered MAPK signaling pathway, which has been confirmed to be associated with VSMC proliferation (13) and hypertrophy (14).

Cortistatin (CST) is a recently detected biological peptide secreted from the cerebral cortex, whose biological function has been found not only in protecting the encephalon, immunologic system and endocranium but also in regulating cardiovascular diseases $(15,16)$. In recent years, studies on the effects of CST on cardiovascular diseases have continued. In 2013, a study showed that CST restrains the proliferation and migration of human VSMCs in atherosclerosis and restenosis (17). It reported that CST inhibits the accumulation of extracellular matrix in abdominal aortic aneurysms (18). Moreover, our experimental team previously proved that CST plays a protective role in suppressing vascular calcification $(16,19)$, myocardial infarction (20) and sepsis (21). We are inquisitive whether CST acts as a protector in hypertension and what cellular and molecular mechanisms may be involved. Accordingly, we hypothesized that CST may suppress Ang II-mediated proliferation and migration of VSMCs, and this effect might be associated with MAPK signaling pathways.

\section{Methods}

\section{Chemicals and reagents}

Rat CST-14 was purchased from Phoenix Pharmaceuticals (Belmont, CA, United States). Ang II was purchased from Tocris Bioscience (Minneapolis, Minnesota, United States). Cell counting kit-8 (CCK8) proliferation assays were purchased from Beyotime (Jiangsu, China). PD98059, SB203580, and SP600125 were obtained from SigmaAldrich (Saint Louis, MO, United States). XMD17-109 was obtained from MedChemExpress (Monmouth Junction, NJ, United States). Rabbit polyclonal anti-proliferating cell nuclear antigen (PCNA), anti- $\beta$-tubulin, anti-ERK1/2, antip-ERK1/2, anti-p38 MAPK, anti-p-p38 MAPK, anti-JNK, anti-p-JNK, anti-ERK5, and anti-p-ERK5 antibodies were purchased from Cell Signaling Technology (Beverly, MA, USA). Rabbit polyclonal anti-osteopontin (OPN) and anti- 
$\alpha$-smooth muscle actin ( $\alpha$-SMA) antibodies were purchased from Abcam (Cambridge, MA, USA).

\section{Culture of rat VSMCs}

Healthy male Wistar rats weighing 100-120 g, whose isolated thoracic aorta tissue was used for VSMC culture, were obtained from the Animal Center laboratory, the Second Affiliated Hospital of Harbin Medical University. The animal care and experimental procedures were approved by the Animal Management Rule of the Ministry of Health, People's Republic of China (document No. 55, 2001) and carried out in accordance with the Ethics Committee of the First Affiliated Hospital of Harbin Medical University, China. In short, after removing the intima and adventitia of an isolated thoracic aorta, the aorta was processed into thin slices with an area of approximately 1-2 $\mathrm{mm}^{3}$, which were first cultured in Dulbecco's modified Eagle's medium (DMEM) (Corning, New York, NY, United States) containing $4.5 \mathrm{~g} / \mathrm{L}$ glucose, $20 \%$ fetal bovine serum (FBS) (Clark, Claymont, DE, United States) and $1 \%$ penicillin-streptomycin solution in an incubation environment of 5\% $\mathrm{CO}_{2}$ at 37 a for approximately 3-7 days. After that, primary VSMCs grown from the periphery of tissue blocks were cultured in the medium (only the FBS concentration changed to $10 \%$, while other components remained unchanged). VSMCs after 4-8 passages were selected for our experiments. For experimental administration, VSMCs were treated with Ang II $\left(10^{-7} \mathrm{M}\right)$ for $24 \mathrm{~h}$ after a $12 \mathrm{~h}$ stationary stage in pure DMEM or were pretreated for $30 \mathrm{~min}$ with CST $\left(10^{-8}\right.$ or $\left.10^{-7} \mathrm{M}\right)$ before Ang II administration. For inhibition assays, PD98059 $\left(5 \times 10^{-5} \mathrm{M}\right)$, SB203580 $\left(10^{-5} \mathrm{M}\right)$, SP600125 $\left(10^{-5} \mathrm{M}\right)$ and XMD17-109 $\left(10^{-6} \mathrm{M}\right)$ pretreatments were added for $1 \mathrm{~h}$ before Ang II administration.

\section{Western blot analysis}

Total protein extracts collected from all treated sample groups were lysed in radioimmunoprecipitation assay (RIPA) buffer with $1 \%$ protease and phosphatase inhibitors (Beyotime, Jiangsu, China) on ice for $30 \mathrm{~min}$, then centrifuged at $13,500 \mathrm{rpm}$ for $20 \mathrm{~min}$ at $4{ }^{\circ} \mathrm{C}$ to obtain the supernatant which was analyzed by a bicinchoninic acid (BCA) protein assay kit (Beyotime, Jiangsu, China). Approximately 10-30 $\mu \mathrm{g}$ of protein extracts were equally added to each electrophoresis lane on 10\% SDS-PAGE gels and then transferred to polyvinylidene fluoride (PVDF) membranes (Corning, New York, NY, United States) with a blocking solution of $5 \%$ nonfat milk powder in Tris-buffered saline containing Tween 20 for $1 \mathrm{~h}$. Target membranes were incubated for $12 \mathrm{~h}\left(4^{\circ} \mathrm{C}\right)$ with respective primary antibodies against PCNA $(1: 1,000), \beta$-tubulin (1:1,000), ERK1/2 (1:1,000), p-ERK1/2 (1:2,000), p38 MAPK $(1: 1,000)$, p-p38 MAPK $(1: 1,000)$, JNK $(1: 1,000)$, JNK (1:1,000), ERK5 (1:1,000), p-ERK5 (1:1,000), OPN $(1: 1,000)$, and $\alpha$-SMA $(1: 1,000)$. Subsequently, the protein bands were detected by incubation with horseradish peroxidase-conjugated secondary antibodies and developed by the electrochemiluminescence (ECL) system.

\section{CCK8 analysis}

CCK8 was used to determine cell proliferation. VSMCs were seeded in a 96-well plate at a density of $2 \times 10^{3}$ cells/well in $100 \mu \mathrm{L}$ of cell solution with $10 \mu \mathrm{L}$ of CCK8 solution for $1.5 \mathrm{~h}$ at $37^{\circ} \mathrm{C}$. The optical density (OD) was examined at $450 \mathrm{~nm}$ with a LI-COR microplate reader (Lincoln, Nebraska, United States). Cell proliferation was calculated by the following formula: cell proliferation = OD values (treated groups)/OD values (control groups).

\section{Transwell cell migration assay}

VSMCs in each group were collected and suspended in pure DMEM, and then the density was adjusted to $2 \times 10^{5}$ cells $/ \mathrm{ml}$. The cell suspensions with or without CST in each group were cultured in the upper chamber of a Transwell $(8.0 \mu \mathrm{m}$, Corning, New York, NY, United States), and at the same time, the lower chamber was filled with DMEM (10\% FBS) with or without Ang II for $24 \mathrm{~h}$. The superior layer cells of the upper chamber were washed with phosphate-buffered saline (PBS) (3 times) and erased with cotton swabs, and then they were fixed in $4 \%$ paraformaldehyde and stained with $0.1 \%$ crystal violet. After rinsing with PBS, the cells were observed and photographed under an inverted microscope. The number of stained cells was counted ( 5 visual fields in each group), and the experiment was repeated at least three times.

\section{Statistical analysis}

Values are expressed as the mean \pm SD, and all experiments were repeated at least 3 times, using GraphPad Prism 5.0 software for analysis. Differences among experimental groups were analyzed using one-way ANOVA followed by 
A
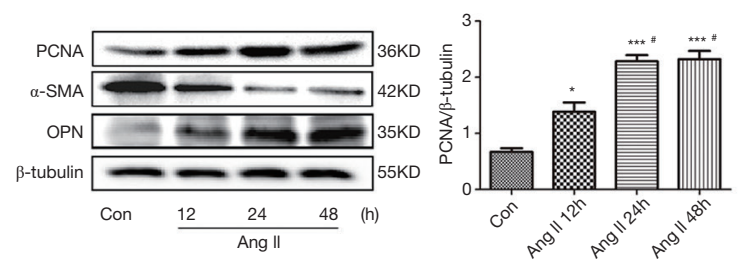

C
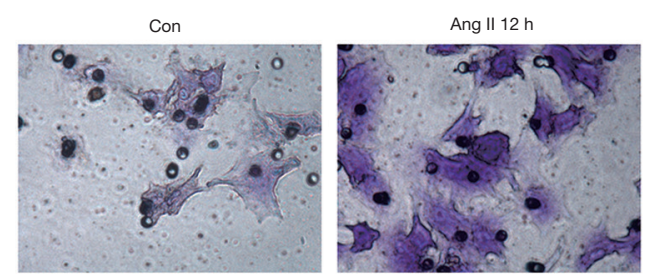
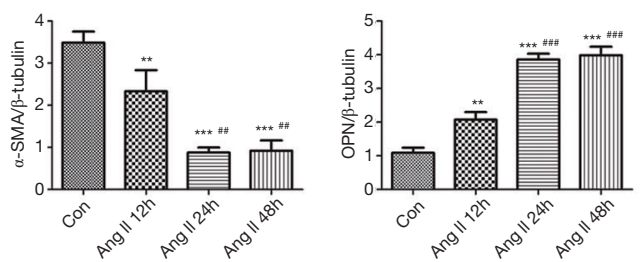

B
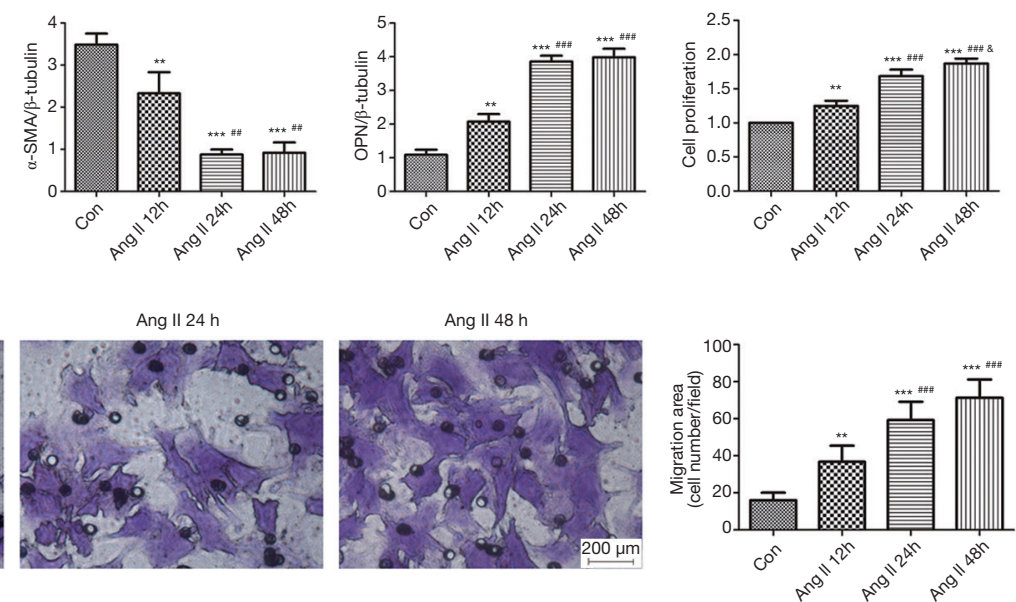

Figure 1 Effects of Ang II on the the proliferation and migration of rat VSMCs at different time points. (A) Western blotting showed increased PCNA and OPN, and decreased $\alpha$-SMA expressions mediated by Ang II at 12, 24 and $48 \mathrm{~h}$ (n=3). (B) CCK8 showed increased cell proliferation rate mediated by Ang II at 12, 24 and 48 h (n=5). (C) Transwell results showed increased VSMC migration mediated by Ang II at

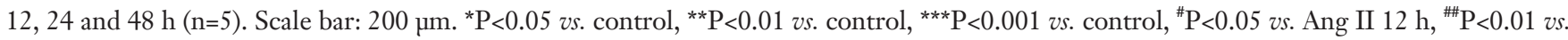
Ang II 12 h, ${ }^{\# \#+~} \mathrm{P}<0.001$ vs. Ang II 12 h, ${ }^{~} \mathrm{P}<0.05$ vs. Ang II 24 h. Con, control group; Ang II, angiotensin II group; VSMC, vascular smooth muscle cell; PCNA, proliferating cell nuclear antigen; OPN, osteopontin; $\alpha$-SMA, $\alpha$-smooth muscle actin; CCK8, cell counting kit-8.

Tukey's test. $\mathrm{P}<0.05$ was regarded as significant.

\section{Results}

\section{Effects of Ang II on VSMC proliferation and migration}

VSMCs were treated with Ang II $\left(10^{-7} \mathrm{M}\right)$ at diverse time points $(0,12,24$ and $48 \mathrm{~h})$. As shown in Figure $1 \mathrm{~A}$, we observed that Ang II stimulated VSMC proliferation, with an increase in the expression levels of PCNA and OPN and a decrease in the expression level of $\alpha$-SMA. The changes in the expression levels of proliferation-related proteins at 24 and $48 \mathrm{~h}$ were more obvious than those at $12 \mathrm{~h}$. Furthermore, CCK8 analysis further showed that Ang II caused an increase in VSMC proliferation with increasing time (Figure 1B). Transwell experiments visibly revealed that Ang II stimulated VSMC migration with increasing time (Figure 1C).

\section{Ang II activated the ERK1/2, p38 MAPK, 7NK and ERK5 signaling pathways in VSMCs}

When VSMCs were treated with Ang II for 0, 12, 24 and $48 \mathrm{~h}$, we observed an obvious increase in the expression levels of p-ERK1/2, p-p38 MAPK, p-JNK and p-ERK5 and an time-dependent increase in the expression level of
p-ERK1/2 under Ang II stimulation (Figure $2 A, B, C, D, E$ ). For the phosphorylated protein expression levels of the other MAPK signaling pathways, there were no large differences between 24 and $48 \mathrm{~h}$ of Ang II treatment in VSMCs. Therefore, in our study, we chose $24 \mathrm{~h}$ as the suitable administration time of Ang II to VSMCs.

\section{Ang II exacerbated VSMC proliferation and migration via the ERK1/2, p38 MAPK, FNK and ERK5 signaling patbways}

To delve deeply into the role of the MAPK signaling pathways in VSMC proliferation and migration promoted by Ang II, we attempted to use inhibitors of the ERK1/2, p38 MAPK, JNK and ERK5 signaling pathways. For pharmacologic inhibition assays, PD98059, SB203580, SP600125 and XMD17-109 pretreatments were independently added $1 \mathrm{~h}$ before Ang II treatment of VSMCs. In Figure $3 A, B, C, D$, we observed that PD98059, SB203580, SP600125, and XMD17-109 clearly blocked the Ang II-mediated increases in protein expression levels of p-ERK1/2, p-p38 MAPK, p-JNK and p-ERK5, respectively. As shown in Figure $3 A, B, C, D$, in VSMCs, PD 98059, SB203580, SP600125 and XMD17-109 individually downregulated the expression levels of PCNA 
A

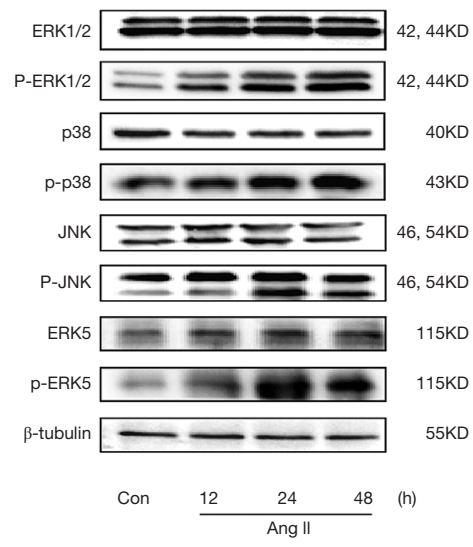

B

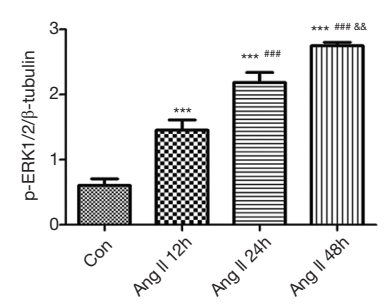

$\mathrm{D}$

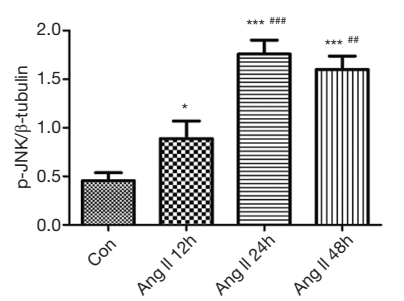

C

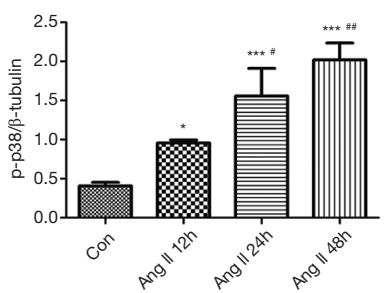

E

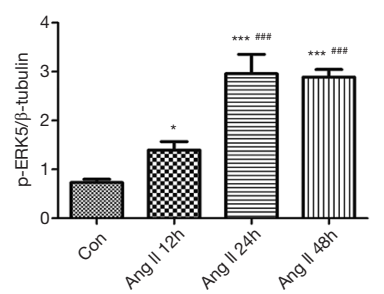

Figure 2 Effects of Ang II on the MAPK family pathways in rat VSMCs at different time points. (A-E) Western blotting showed increased p-ERK1/2, p-p38 MAPK, p-JNK and p-ERK5 expressions mediated by Ang II at 12, 24 and $48 \mathrm{~h}(\mathrm{n}=5)$. ${ }^{*} \mathrm{P}<0.05$ vs. control, ${ }^{* * *} \mathrm{P}<0.001$ vs. control, ${ }^{\#} \mathrm{P}<0.05$ vs. Ang II $12 \mathrm{~h},{ }^{\# \#} \mathrm{P}<0.01$ vs. Ang II $12 \mathrm{~h},{ }^{{ }^{* \prime \prime}} \mathrm{P}<0.001$ vs. Ang II $12 \mathrm{~h},{ }^{{ }^{\& \&}} \mathrm{P}<0.01$ vs. Ang II $24 \mathrm{~h}$. Con, control group; Ang II, angiotensin II group; VSMC, vascular smooth muscle cell; MAPK, mitogen-activated protein kinase; ERK, extracellular signal-regulated kinase; JNK, c-Jun N-terminal kinase.

and OPN that were increased by Ang II and upregulated the expression level of $\alpha$-SMA that was decreased by Ang II. Moreover, in agreement with the western blot results, CCK8 analysis showed that administration of the inhibitors attenuated Ang II-induced cell proliferation (Figure 3E). Transwell observation further corroborated that PD98059, SB203580, SP600125, and XMD17-109 mitigated the migration of VSMCs stimulated by Ang II (Figure 3F). These results indicated that the four main MAPK signaling pathways (ERK1/2, p38 MAPK, JNK and ERK5) may all be involved in Ang II-promoted proliferation and migration.

\section{CST inbibited Ang II-stimulated VSMC proliferation and migration and the activations of ERK1/2, p38 MAPK, FNK and ERK5 signaling patbways}

To explore the potential role of CST in VSMC proliferation and migration promoted by Ang II and to further study the possible mechanism involved, we pretreated VSMCs with CST at concentrations of $10^{-8}$ and $10^{-7} \mathrm{M}$, followed by Ang II stimulation. Then, we detected the effects of CST on the proliferation and migration of VSMCs promoted by Ang II and whether CST had an effect on the MAPK family signaling pathways activated by Ang II. As shown in Figure $4 A$, both $10^{-8}$ and $10^{-7} \mathrm{M}$ CST positively downregulated the protein expression levels of PCNA and OPN and simultaneously upregulated that of $\alpha$-SMA. From a CCK8 test, we observed that CST pretreatment decreased the proliferation rate induced by Ang II in VSMCs (Figure 4B). Moreover, compared with the concentration of $10^{-8} \mathrm{M}, 10^{-7}$ $M$ could more obviously suppress Ang II-induced VSMC proliferation (Figure 4A,B). Transwell observation showed that CST at a concentration of $10^{-7} \mathrm{M}$ refrained the VSMC migration induced by Ang II but $10^{-8} \mathrm{M}$ had no obvious effect on Ang II-mediated cell migration (Figure $4 C$ ).

As shown in Figure $4 A$, pretreatment with CST downregulated the expression levels of p-ERK1/2, p-p38 MAPK, p-JNK and p-ERK5 that were stimulated by Ang II. In a detailed comparison, there was no large difference between the effects of the concentrations of $10^{-8}$ and $10^{-7} \mathrm{M}$ of CST on the expression level of p-ERK1/2. However, for the expression levels of p-p38 MAPK, JNK and p-ERK5, there remained an obvious significant difference between $10^{-8}$ and $10^{-7} \mathrm{M}$ CST. CST at the concentration of $10^{-8} \mathrm{M}$ did not downregulate the expression level of p-JNK, but $10^{-7} \mathrm{M}$ clearly did. In conclusion, CST at a concentration of $10^{-7} \mathrm{M}$ was more impactful than $10^{-8} \mathrm{M}$ CST to inhibit the proliferation and migration of VSMCs and the activation of the MAPK signaling pathways under Ang II conditions. These findings indicated that CST exerts the inhibitory function on Ang II-induced proliferation and migration in VSMCs, which might occur possibly via inactivating the 

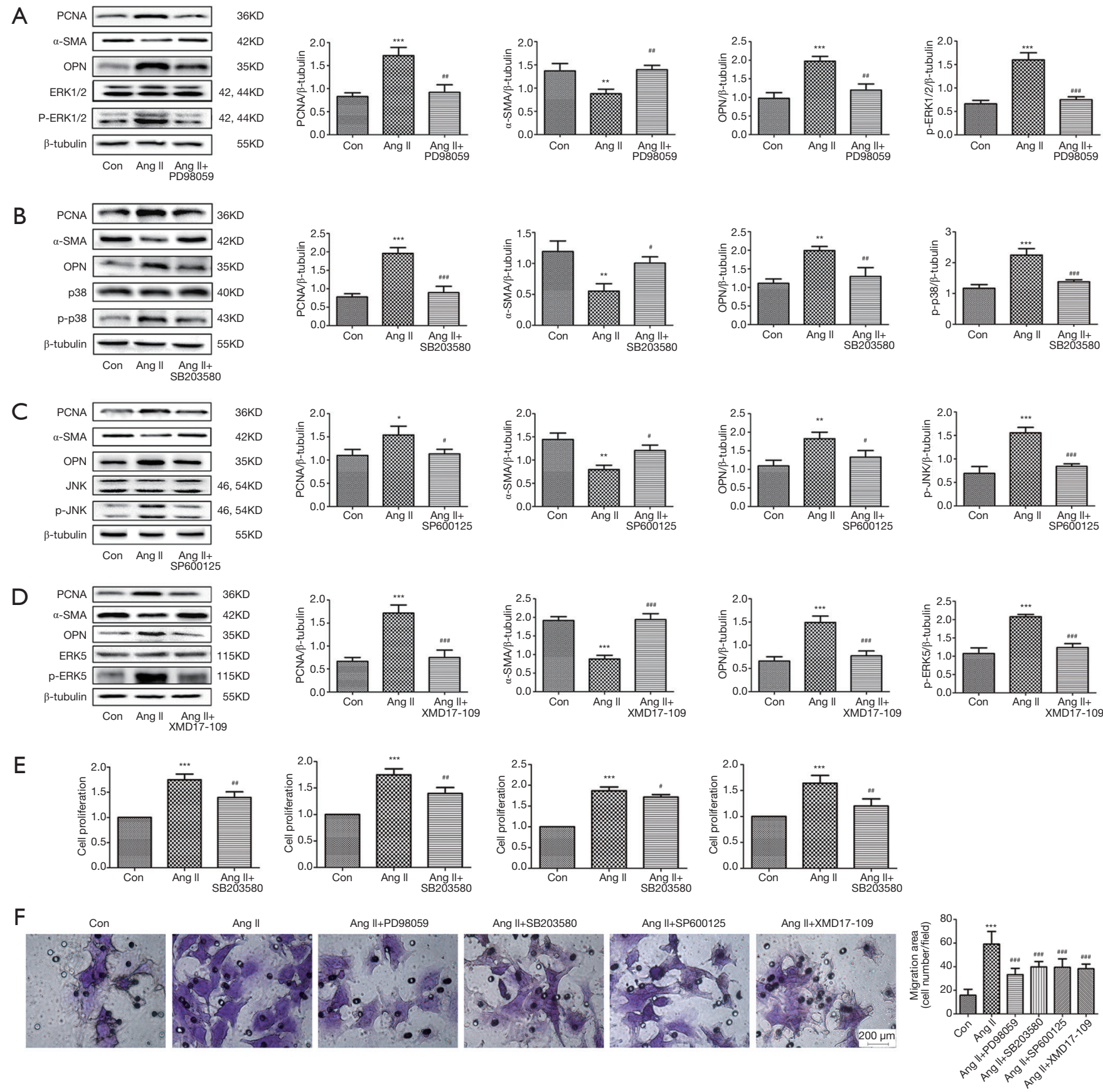

Figure 3 The inhibitors of ERK1/2, p38 MAPK, JNK and ERK5 signaling pathways (PD98059, SB203580, SP600125 and XMD17109) suppress Ang II-mediated rat VSMC proliferation and migration. (A-D) Western blotting detected the expression levels of PCNA, $\alpha$-SMA, OPN, ERK1/2, p-ERK1/2, p38 MAPK, p-p38 MAPK, JNK, p-JNK, ERK5 and p-ERK5 in VSMCs (n=4). (E) CCK8 showed that PD98059, SB203580, SP600125 and XMD17-109 respectively suppressed the cell proliferation rate mediated by Ang II (n=4). (F) Transwell results showed that PD98059, SB203580, SP600125 and XMD17-109 inhibited Ang II-mediated VSMC migration (n=5). Scale bar: $200 \mu \mathrm{m}$. ${ }^{*} \mathrm{P}<0.05$ vs. control, ${ }^{* *} \mathrm{P}<0.01$ vs. control, ${ }^{* * *} \mathrm{P}<0.001$ vs. control, ${ }^{*} \mathrm{P}<0.05$ vs. Ang II, ${ }^{\# \#} \mathrm{P}<0.01$ vs. Ang II, ${ }^{\text {"\#\#! }} \mathrm{P}<0.001$ vs. Ang II. Con, control group; Ang II, angiotensin II group; MAPK, mitogen-activated protein kinase; ERK, extracellular signal-regulated kinase; JNK, c-Jun N-terminal kinase; VSMC, vascular smooth muscle cell; PCNA, proliferating cell nuclear antigen; OPN, osteopontin; $\alpha$-SMA, $\alpha$-smooth muscle actin; CCK8, cell counting kit-8. 

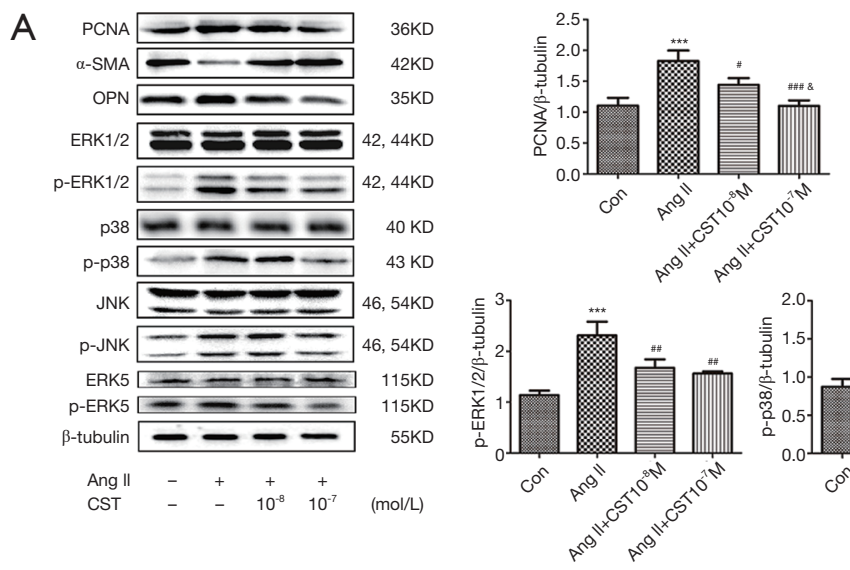
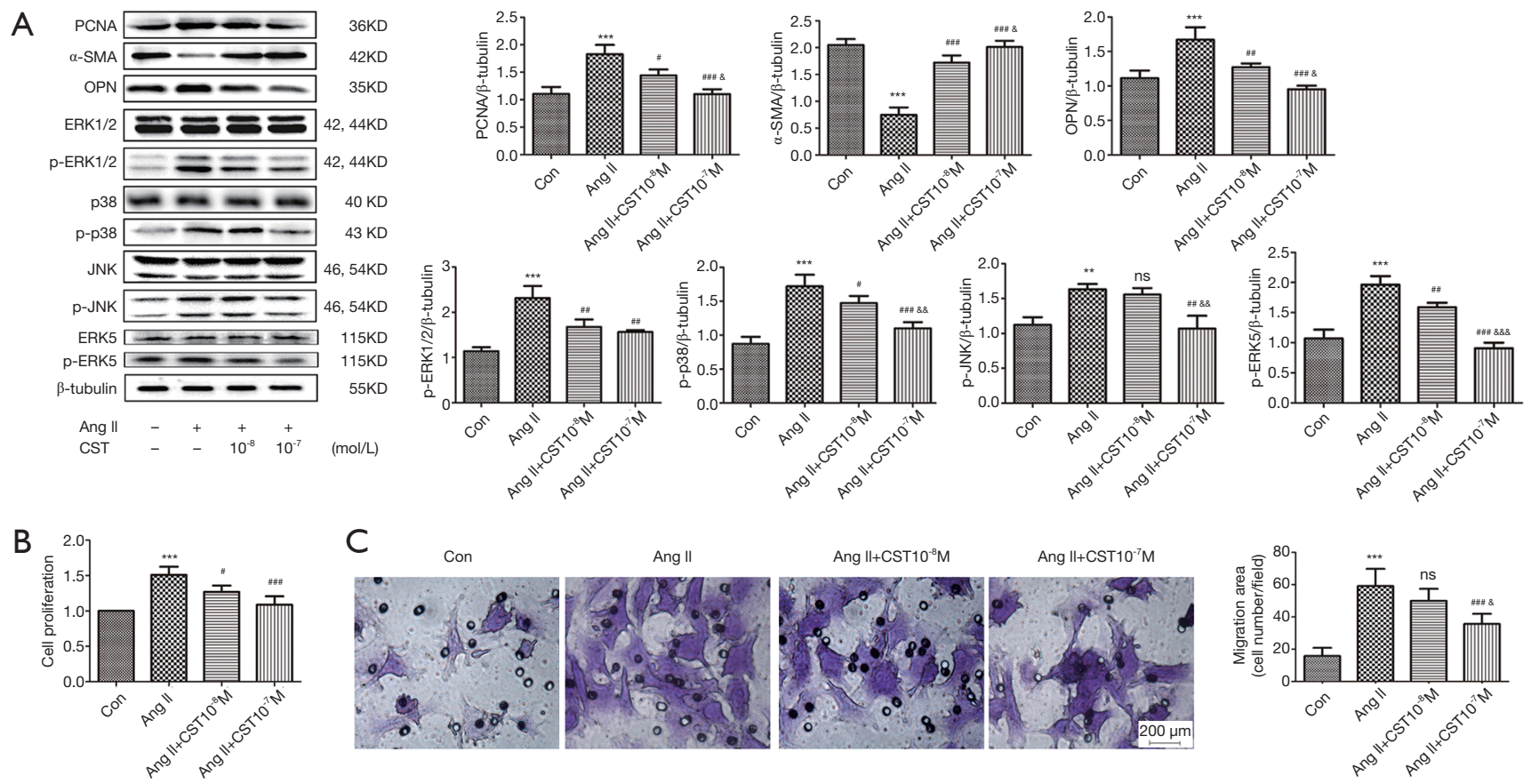

Figure 4 CST attenuates rat VSMC proliferation and migration mediated by Ang II via the MAPK family pathways. (A) Western blotting detected the expression levels of PCNA, $\alpha$-SMA, OPN, ERK1/2, p-ERK1/2, p38 MAPK, p-p38 MAPK, JNK, p-JNK, ERK5 and p-ERK5 in VSMCs ( $\mathrm{n}=4)$. (B) CCK8 showed that $10^{-8}$ and $10^{-7}$ M CST suppressed the cell proliferation rate mediated by Ang II (n=4). (C) Transwell results showed that $10^{-7} \mathrm{M}$ rather than $10^{-8} \mathrm{M}$ CST inhibited Ang II-induced VSMC migration, $(\mathrm{n}=5)$. Scale bar: $200 \mu \mathrm{m} .{ }^{* *} \mathrm{P}<0.01$ vs. control, ${ }^{* *} \mathrm{P}<0.001$ vs. control, ${ }^{\#} \mathrm{P}<0.05$ vs. Ang II, ${ }^{\# \#} \mathrm{P}<0.01$ vs. Ang II, ${ }^{\# \#} \mathrm{P}<0.001$ vs. Ang II, ${ }^{*} \mathrm{P}<0.05$ vs. Ang II + CST $10^{-8} \mathrm{M},{ }^{\text {\&\& }} \mathrm{P}<0.01$ vs. Ang II + CST $10^{-8} \mathrm{M},{ }^{\& \& \&} \mathrm{P}<0.001$ vs. Ang II + CST $10^{-8} \mathrm{M}$. Con, control group; Ang II, angiotensin II group; CST, cortistatin; MAPK, mitogen-activated protein kinase; ERK, extracellular signal-regulated kinase; JNK, c-Jun N-terminal kinase; VSMC, vascular smooth muscle cell; PCNA, proliferating cell nuclear antigen; OPN, osteopontin; $\alpha$-SMA, $\alpha$-smooth muscle actin; CCK8, cell counting kit-8.

MAPK signaling pathways.

\section{Discussion}

Our study uncovered a protective role of CST in Ang IImediated proliferation and migration of VSMCs. We also obtained the result that Ang II induced the proliferation and migration of VSMCs, and we observed that 24 and $48 \mathrm{~h}$ of Ang II administration could visibly stimulate cell proliferation and migration. Then, we detected activation of the MAPK signaling pathways, namely, the ERK1/2, p38 MAPK, JNK and ERK5 pathways, during Ang II stimulation of VSMCs. Mechanistically, pharmacological inhibitors of these signaling pathways separately suppressed the Ang II-induced proliferation and migration of VSMCs. Finally, we discovered that CST suppressed the proliferation and migration of VSMCs stimulated by Ang II as well as the activation of the MAPK signaling pathways, indicating that the ERK1/2, p38 MAPK, JNK and ERK5 signaling pathways may be involved in the restraining effect of CST against Ang II-induced proliferation and migration of VSMCs.

It is generally accepted that Ang II can activate diverse signaling pathways by which it controls the proliferation and migration of VSMCs, which is the central link of vascular remodeling and morbidity leading to hypertension, arteriosclerosis and other cardiovascular diseases. Among the multitudinous signaling pathways, the MAPK family pathways act as a prominent participant in Ang II-mediated cell proliferation, differentiation, migration, apoptosis and other cell pathological changes. Previous experimental studies have investigated the regulatory role of MAPK in the Ang II-mediated VSMC model. However, there is no systematic exhaustive research on the regulatory role of the classic members of the MAPK family pathways in Ang IIpromoted VSMC proliferation and migration. Consistent 
with previous studies on the ERK1/2 and JNK pathways, our data showed that Ang II activates phosphorylation of the ERK1/2 and JNK in VSMCs at successive time points (Figure $2 A, B, D$ ). Inconsistently, as for whether p38 MAPK controls cell proliferation, disagreement exists. Some studies have reported that Ang II causes VSMC proliferation and migration via the p38 MAPK pathway $(10,22,23)$. Those results showed that upregulation of the p38 MAPK inhibits high sodium-stimulated VSMC proliferation (24), implying that transduction of the p38 MAPK may not be the same in response to different inducements in VSMCs. However, a study reported that the p38 MAPK decreases Ang II-stimulated cell growth by downregulating activation of ERK1/2 and that the drug inhibitors of p38 MAPK suppress the Ang II-mediated p38 MAPK activation, but promote Ang II-mediated ERK1/2 activation, suggesting that this discrepant result may be due to the interaction between diverse signaling pathways (25). But in our study, we found that Ang II upregulated p38 MAPK and induced cell proliferation and migration, which were partly reversed by an inhibitor of p38 MAPK, SB203580, suggesting that activation of the p38 MAPK could promote the proliferation and migration of VSMCs mediated by Ang II (Figure 2A,C and Figure 3B,E,F). Nevertheless, interactions among the MAPK family pathways in the progression of cell proliferation and migration mediated by Ang II require further explorations. ERK5, the most recently discovered, nonclassical member of the MAPK family pathways, has been confirmed to be related to cell inflammation (26), hypertrophy (14), proliferation $(13,27)$ and migration (28). Our data showed that ERK5 is associated with VSMC proliferation and migration stimulated by Ang II, which were partly attenuated when XMD17-109 (an inhibitor of ERK5) was used (Figure 2A,E and Figure 3D,E,F). Generally, our results demonstrated that all the MAPK family pathways, namely, ERK1/2, p38 MAPK, JNK, and ERK5, could be involved in Ang II-mediated proliferation and migration, which may all promote regulatory responsiveness to the proliferation and migration of VSMCs. To our knowledge, the four MAPK pathways might share overlapping moderation effects on Ang IIpromoted proliferation and migration of VSMCs. Whether a mutually positive or negative interaction mechanism in the MAPK family pathways exists requires deeper research.

A previous study certified that CST exerts an inhibitory capability on atherosclerosis and restenosis (17) via suppression of abnormal VSMC proliferation and migration. However, whether CST also exhibits a protective function against hypertension as well as vascular remodeling, specifically VSMC proliferation and migration, has not been thoroughly investigated. In our study, we showed that the proliferation and migration of VSMCs under Ang II stimulation could be alleviated by CST at a preferred concentration of $10^{-7} \mathrm{M}$ (Figure 4). However, how CST determines its protective effect on proliferation and migration of VSMCs promoted by Ang II and the possible molecular mechanism participating remains unclear. CST, which binds to somatostatin (sst2 and sst5) and ghrelin receptors, was proven to be related to the cAMP, p38 MAPK and Akt signaling pathways in platelet-derived growth factor (PDGF)-induced human aortic smooth muscle cells (17). We previously demonstrated that CST is associated with the GSK3 $\beta / \beta$-catenin signaling pathway in reducing aortic calcification (16). In abdominal aortic aneurysms, CST decreases the expression levels of MMP2 and MMP9 by downregulating the ERK1/2 signaling pathway in vivo and in vitro (18). In consideration of the above-mentioned MAPK family pathways involved in Ang II-mediated VSMC proliferation and migration, we speculated whether CST could reduce the proliferation and migration of VSMCs possibly by the inactivating the MAPK family pathways. Hence, we found that CST downregulated the phosphorylation of ERK1/2, p38 MAPK, JNK, and ERK5 stimulated by Ang II in VSMCs, illustrating that CST suppresses Ang II-promoted proliferation and migration, in which the activation of the MAPK family pathways might be involved.

\section{Conclusions}

To summarize, we render the first evidence for a connection between CST and all the MAPK family pathways because CST exerted its suppressive function on Ang II-mediated proliferation and migration of VSMCs possibly by partially deactivating the ERK1/2, p38 MAPK, JNK, ERK5 pathways revealing that the activation of the four pathways may be involved in the proliferation and migration of VSMCs stimulated by Ang II. Therefore, CST could be a preeminently preventive factor for the proliferation and migration of VSMCs and a prospective therapeutic target for vascular remodeling and hypertension.

\section{Acknowledgments}

Funding: Our research was supported by the National Natural Science Foundation of China (No. 81370319, 
81200235 and 81570437).

\section{Footnote}

Conflicts of Interest: The authors have no conflicts of interest to declare.

Ethical Statement: The authors are accountable for all aspects of the work in ensuring that questions related to the accuracy or integrity of any part of the work are appropriately investigated and resolved. The animal care and experimental procedures were approved by the Animal Management Rule of the Ministry of Health, People's Republic of China (document No. 55, 2001) and carried out in accordance with the Ethics Committee of the First Affiliated Hospital of Harbin Medical University, China.

\section{References}

1. Li H, Liu X, Zhang L, et al. LncRNA BANCR facilitates vascular smooth muscle cell proliferation and migration through JNK pathway. Oncotarget 2017;8:114568-75.

2. Mehta PK, Griendling KK. Angiotensin II cell signaling: physiological and pathological effects in the cardiovascular system. Am J Physiol Cell Physiol 2007;292:C82-97.

3. Jia G, Stormont RM, Gangahar DM, et al. Role of matrix Gla protein in angiotensin II-induced exacerbation of vascular calcification. Am J Physiol Heart Circ Physiol 2012;303:H523-32.

4. Shi N, Chen SY. Mechanisms simultaneously regulate smooth muscle proliferation and differentiation. J Biomed Res 2014;28:40-6.

5. Taniyama Y, Ushio-Fukai M, Hitomi H, et al. Role of p38 MAPK and MAPKAPK-2 in angiotensin II-induced Akt activation in vascular smooth muscle cells. Am J Physiol Cell Physiol 2004;287:C494-9.

6. Kyotani Y, Zhao J, Tomita S, et al. Olmesartan inhibits angiotensin II-Induced migration of vascular smooth muscle cells through Src and mitogen-activated protein kinase pathways. J Pharmacol Sci 2010;113:161-8.

7. Feng W, Zhang K, Liu Y, et al. Apocynin attenuates angiotensin II-induced vascular smooth muscle cells osteogenic switching via suppressing extracellular signalregulated kinase 1/2. Oncotarget 2016;7:83588-600.

8. Su J, Xu HT, Yu JJ, et al. Luteolin Ameliorates Hypertensive Vascular Remodeling through Inhibiting the Proliferation and Migration of Vascular Smooth Muscle Cells. Evid Based Complement Alternat Med
2015;2015:364876.

9. Nagayama K, Kyotani Y, Zhao J, et al. Exendin-4 Prevents Vascular Smooth Muscle Cell Proliferation and Migration by Angiotensin II via the Inhibition of ERK1/2 and JNK Signaling Pathways. PLoS One 2015;10:e0137960.

10. Won SM, Park YH, Kim HJ, et al. Catechins inhibit angiotensin II-induced vascular smooth muscle cell proliferation via mitogen-activated protein kinase pathway. Exp Mol Med 2006;38:525-34.

11. Force T, Pombo CM, Avruch JA, et al. Stress-activated protein kinases in cardiovascular disease. Circ Res 1996;78:947-53.

12. Kudoh S, Komuro I, Mizuno T, et al. Angiotensin II stimulates c-Jun NH2-terminal kinase in cultured cardiac myocytes of neonatal rats. Circ Res 1997;80:139-46.

13. Zhao M, Liu Y, Bao M, et al. Vascular smooth muscle cell proliferation requires both p38 and BMK1 MAP kinases. Arch Biochem Biophys 2002;400:199-207.

14. Zhao Z, Geng J, Ge Z, et al. Activation of ERK5 in angiotensin II-induced hypertrophy of human aortic smooth muscle cells. Mol Cell Biochem 2009;322:171-8.

15. Liu Y, Yin XH, Qi YF. Progress in biological effects of cortistatin. Sheng Li Ke Xue Jin Zhan 2009;40:219-24.

16. Liu Y, Lin F, Fu Y, et al. Cortistatin inhibits arterial calcification in rats via GSK3beta/beta-catenin and protein kinase C signalling but not c-Jun N-terminal kinase signalling. Acta Physiol (Oxf) 2018;223:e13055.

17. Duran-Prado M, Morell M, Delgado-Maroto V, et al. Cortistatin inhibits migration and proliferation of human vascular smooth muscle cells and decreases neointimal formation on carotid artery ligation. Circ Res 2013;112:1444-55.

18. Chai H, Tao Z, Chen W, et al. Cortistatin attenuates angiotensin II-induced abdominal aortic aneurysm through inactivation of the ERK1/2 signaling pathways. Biochem Biophys Res Commun 2018;495:1801-6.

19. Liu Y, Lin F, Fu Y, et al. Cortistatin inhibits calcification of vascular smooth muscle cells by depressing osteoblastic differentiation and endoplasmic reticulum stress. Amino Acids 2016;48:2671-81.

20. Shi ZY, Liu Y, Dong L, et al. Cortistatin Improves Cardiac Function After Acute Myocardial Infarction in Rats by Suppressing Myocardial Apoptosis and Endoplasmic Reticulum Stress. J Cardiovasc Pharmacol Ther 2017;22:83-93.

21. Zhang B, Liu Y, Zhang JS, et al. Cortistatin protects myocardium from endoplasmic reticulum stress induced apoptosis during sepsis. Mol Cell Endocrinol 
2015;406:40-8.

22. Shen YJ, Zhu XX, Yang X, et al. Cardamonin inhibits angiotensin II-induced vascular smooth muscle cell proliferation and migration by downregulating $\mathrm{p} 38$ MAPK, Akt, and ERK phosphorylation. J Nat Med 2014;68:623-9.

23. Sheng-Long C, Yan-Xin W, Yi-Yi H, et al. AVE0991, a Nonpeptide Compound, Attenuates Angiotensin IIInduced Vascular Smooth Muscle Cell Proliferation via Induction of Heme Oxygenase-1 and Downregulation of p-38 MAPK Phosphorylation. Int J Hypertens 2012;2012:958298.

24. Wu Y, Zhou J, Wang H, et al. The activation of p38 MAPK limits the abnormal proliferation of vascular smooth muscle cells induced by high sodium concentrations. Int J Mol Med 2016;37:74-82.

Cite this article as: Wang Y, Zhang X, Gao L, Li J, Chen W, Chi J, Zhang X, Fu Y, Zhao M, Liu N, Li Y, Xu Y, Yang K, Yin X, Liu Y. Cortistatin exerts antiproliferation and antimigration effects in vascular smooth muscle cells stimulated by Ang II through suppressing ERK1/2, p38 MAPK, JNK and ERK5 signaling pathways. Ann Transl Med 2019;7(20):561. doi: 10.21037/atm.2019.09.45
25. Kintscher U, Bruemmer D, Blaschke F, et al. p38 MAP kinase negatively regulates angiotensin II-mediated effects on cell cycle molecules in human coronary smooth muscle cells. Biochem Biophys Res Commun 2003;305:552-6.

26. Wilhelmsen K, Xu F, Farrar K, et al. Extracellular signalregulated kinase 5 promotes acute cellular and systemic inflammation. Sci Signal 2015;8:ra86.

27. Sharma G, Goalstone ML. Regulation of ERK5 by insulin and angiotensin-II in vascular smooth muscle cells. Biochem Biophys Res Commun 2007;354:1078-83.

28. Izawa Y, Yoshizumi M, Ishizawa K, et al. Big mitogenactivated protein kinase 1 (BMK1)/extracellular signal regulated kinase 5 (ERK5) is involved in platelet-derived growth factor (PDGF)-induced vascular smooth muscle cell migration. Hypertens Res 2007;30:1107-17. 